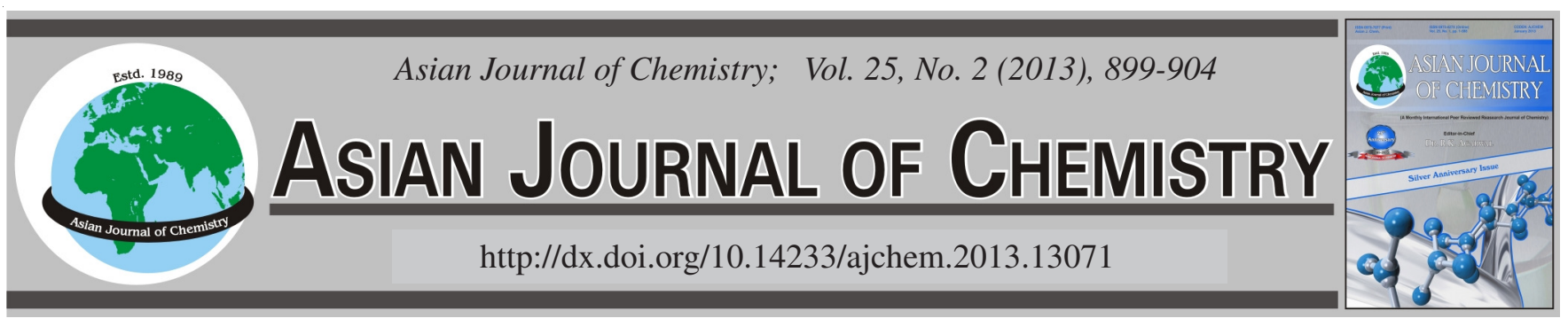

\title{
A Recyclable and Highly Effective Sulfamic Acid/EtOH Catalytic System in Synthesis and Natural Product Chemistry of Lignan Compounds
}

\author{
Z. Ali ${ }^{*}$, S. DEO and F. INAM
}

Department of Chemistry, Institute of Science, R.T. Road, Nagpur-440 001, India

*Corresponding author: E-mail: tanishqme@rediffmail.com

The utilization of green chemistry techniques is dramatically reducing chemical waste and reaction times and has recently been proven in several organic syntheses and chemical transformations. To illustrate these advantages sulphamic acid is used as a novel recyclable heterogeneous catalyst in the cyclization of Perkin condensation product $\alpha$-arylidine- $\beta$-benzoyl propionic acid to achieve biologically potent 1-phenylnaphthalene system and pericarbonyl lactone lignans under two experimental conditions i.e., conventional and microwave irradiation. The generality of this protocol has been demonstrated by synthesizing a variety of substituted 1-phenyl-naphthalene systems in excellent yields, short reaction time and with good purity.

Key Words: Sulphamic acid/Ethanol system, Lignans, Cyclization, Recyclable system.

ᄂ - - - - - - - - - - - - - - - -

\section{INTRODUCTION}

1-Phenylnaphthalene system attracted special attention of organic chemists because these are biologically active with potential applications as antiinflammatory ${ }^{1}$, antibacterial ${ }^{2}$, antioxidant $^{3}$, anticancer ${ }^{4}$ and CNS depressants ${ }^{5}$. 1-Phenylnaphthalenes are versatile moieties in that their pendant like skeleton exists in a number of pharmaceuticals and natural products. As a result of their importance from industrial, biological and synthetic point of view, it is desirable to choose an efficient and versatile protocol for their easy and clean synthesis.

The catalytic activity of sulphamic acid (SA) has emerged as a useful acid imparting high region and chemo selectivity in various chemical transformations ${ }^{6-9}$. It is a dry, nonvolatile, non hygroscopic, odorless and white crystalline solid Bronsted acid with outstanding physical properties. It is inexpensive, insoluble in common organic solvents, highly stable and its zwitterionic property makes its recycling and reuse very convenient $^{10,11}$.

We report herein a highly efficient procedure for the synthesis of various substituted 1-phenylnaphthalene systems and pericarbonyl lactones using sulphamic acid as an efficient and versatile catalyst under both methods-conventional and microwave irradiation.

By the use of microwave in combination with sulphamic acid, the term "green chemistry" ideally disappears as all chemistry becomes green.

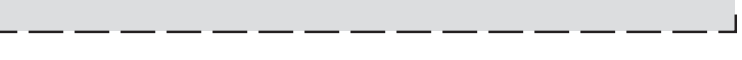

\section{EXPERIMENTAL}

All the chemicals and reagents were of LR or AR grade and procured from S.D. Fine Chem. Limited, (Mumbai), LOBA Chemie, Mumbai, E. Merck (India ) Ltd. and used as received. IR spectra were recorded on Perkin-Elmer FT spectrophotometer in $\mathrm{KBr}$ disc. ${ }^{1} \mathrm{H}$ NMR were recorded on Varian $300 \mathrm{MHz}$ spectrophotometer in $\mathrm{CDCl}_{3}$ as a solvent and TMS as an internal standard. Melting points were recorded on a melting point apparatus with capillary tubes and are uncorrected. Analytical TLC was performed on glass slides coated of silica gel G/UV-254 of $0.2 \mathrm{~mm}$ thickness. Elemental analysis data were recorded using Thermo Finnegan FLASH EA 1112 $\mathrm{CHN}$ analyzer. For the microwave irradiation experiments described below, a conventional (unmodified) house hold microwave oven equipped with a turn able was used (LG Smart Chef MS-255r operating at $2450 \mathrm{MHz}$ having maximum output of $800 \mathrm{~W}$ ).

Conversion of $\beta$-benzoyl propionic acid to $\alpha$-arylidine$\boldsymbol{\beta}$-benzoyl propionic acid: For the synthesis of 1-phenylnaphthalene system, $\beta$-benzoyl propionic acid was taken as a starting moiety. Perkin condensation of $\beta$-benzoyl propionic acid with aryl aldehyde yields butenolide ${ }^{12}$ which on cleavage by methanolic solution of $\mathrm{Na}_{2} \mathrm{CO}_{3}$ leads to $\alpha$-arylidine- $\beta$ benzoyl propionic acid ${ }^{13}$ (Scheme-I). The system (4) thus contains the required skeleton to prepare 1-phenylnaphthalene system and pericarbonyl lactone lignans ${ }^{14,15}$ (Scheme-II). 
<smiles>O=C1CCC(=O)c2ccccc21</smiles>

$\beta$-benzoyl propionic acid (1)<smiles>[R]c1cc(C=O)cc([R])c1[R]</smiles>

aryl aldehyde (2)
Perkin Reaction

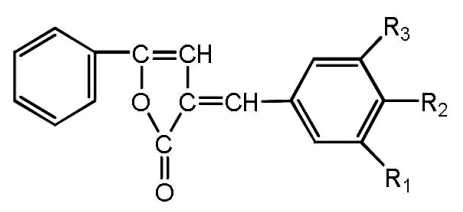

$\alpha$-arylidine $-\gamma$-phenyl - $\Delta, \beta$-butenolide (3)

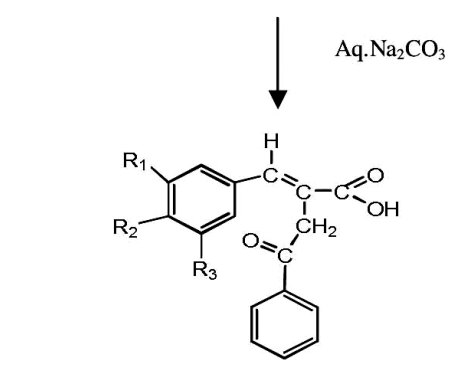

$\alpha$-arylidine - $\beta$-benzoyl propionic acid (4)

Scheme-I

\section{Path-A}

Cyclization of $\alpha$-arylidine- $\beta$-benzoyl propionic acid (4) to 1-phenylnaphthoic acid (7)

Conventional method: A mixture of $1 \mathrm{mmol}$ of $\alpha$-arylidine$\beta$-benzoyl propionic acid, $0.2 \mathrm{mmol}(20 \mathrm{mmol} \%)$ sulphamic acid and ethanol (taken in excess: $10 \mathrm{~mL}$ ) was taken as a reaction solvent in $250 \mathrm{~mL}$ round bottom flask. Stir the mixture vigorously by keeping in reflux at $118-120^{\circ} \mathrm{C}$ for an appropriate time as mentioned in Table-1. After completion of reaction as indicated by TLC, the reaction mixture was cooled at room temperature and diluted with diethyl ether $[3 \times 10 \mathrm{~mL}]$ to precipitate sulphamic acid. Thus sulphamic acid could be separated easily. The combined organic layers were dried over anhydrous $\mathrm{Na}_{2} \mathrm{SO}_{4}$. The solvent was removed and the residue was column chromatographed using petroleum ether:ethyl acetate $(2: 3)$ as the eluent, to obtain pure compound.

Microwave irradiation: The reaction mixture as specified above was taken in a dry $250 \mathrm{~mL}$ beaker. It was mixed properly with the help of glass rod. An inverted funnel was placed over the rim of the beaker and irradiated in a microwave oven at $800 \mathrm{~W}$ for $14-15 \mathrm{~min}$ while monitoring the reaction with the help of TLC. After the completion of the reaction, immediate temperature of the reaction mixture was taken out by the thermometer, which is recorded as $75{ }^{\circ} \mathrm{C}$. The reaction was worked-up and purified as described above under conventional method.
Adopting the above described two alternative methods, four different substituted 1-phenylnaphthoic acids (7a-7d) were identified and characterized (Table-2).

Path-B

Conversion of $\alpha$-arylidine $\beta$-benzoyl propionic acid (4) to $\alpha$-arylidine- $\beta$-methylene- $\beta$-benzoyl propionic acid (5)

To a solution of $\alpha$-arylidine- $\beta$-benzoyl propionic acid in $10 \%$ aq. $\mathrm{NaOH}$ solution was added $40 \%$ formalin solution and was left overnight. It was then cooled and dried with anhydrous sodium sulphate. Ethanol is added to separate the product. Evaporation of solvent gave a gummy solid which was crystallized from ethanol/water to give $\alpha$-arylidine- $\beta$ methylene- $\beta$-benzoyl propionic acid. The IR spectra of (5) showed broad peak at $1660 \mathrm{~cm}^{-1}$ for carboxyl and keto group.

Cyclization of $\alpha$-arylidine- $\beta$-methylene- $\beta$-benzoyl propionic acid (5) to 1-phenyl naphthalene lactone/pericarbonyl lactone $(8)$

Conventional method: A mixture of $1 \mathrm{mmol}$ of $\alpha$-arylidine$\beta$-benzoyl propionic acid, $0.2 \mathrm{mmol}(20 \mathrm{mmol} \%)$ sulphamic acid and ethanol $10 \mathrm{~mL}$ was taken as a reaction solvent and stir the mixture vigorously (using magnetic stirrer) by keeping in reflux at $118-120^{\circ} \mathrm{C}$ for an appropriate time (Table-1). The reaction was worked-up and purified as described above (Path-A).

Microwave irradiation: The reaction mixture as specified above was taken in a dry $250 \mathrm{~mL}$ beaker. It was mixed properly with the help of glass rod. An inverted funnel was placed over the rim of the beaker and irradiated in a microwave oven at $800 \mathrm{~W}$ for $14-15 \mathrm{~min}$ while monitoring the reaction with the help of TLC. After the completion of the reaction, immediate temperature of the reaction mixture was taken out by the thermometer, which is recorded as $75^{\circ} \mathrm{C}$. The reaction was worked-up and purified to give 1-phenyl-naphthalene lactone/ pericarbonyl lactone. Adopting the above described two alternative methods, four different substituted 1-phenyl naphthalene lactones (8a-8d) were identified and characterized (Table-2). Path-C

Conversation of $\alpha$-arylidine- $\beta$-benzoyl propionic acid (4) to methyl- $\alpha$-arylidine- $\beta$-benzoyl propionic acid (6): $\alpha$-Arylidine- $\beta$-benzoyl propionic acid was converted to its ester by $\mathrm{CH}_{2} \mathrm{~N}_{2}$ to give methyl- $\alpha$-arylidine- $\beta$-benzoyl propionic acid having melting point of $96^{\circ} \mathrm{C}$. Its IR spectra showed absorption at $1680 \mathrm{~cm}^{-1}$.

Cyclization of methyl $\alpha$-arylidine- $\beta$-benzoyl propionic acid (6) to 1-phenyl naphthoate (9)

Conventional method: A mixture of $1 \mathrm{mmol}$ of $\alpha$-arylidine$\beta$-methylene- $\beta$-benzoyl propionic acid, $0.2 \mathrm{mmol}$ ( $20 \mathrm{mmol} \%$ ) sulphamic acid and ethanol $10 \mathrm{~mL}$ was taken as a reaction solvent and stir the mixture vigorously (using magnetic stirrer) by keeping in reflux at $118-120{ }^{\circ} \mathrm{C}$ for an appropriate time (Table-1). The reaction was worked-up and purified (likewise Path-A) to give 1-phenylnaphthoate.

Microwave irradiation: The reaction mixture as specified above was taken in a dry $250 \mathrm{~mL}$ beaker. It was mixed properly with the help of glass rod. An inverted funnel was placed over the rim of the beaker and irradiated in a microwave oven at $800 \mathrm{~W}$ for $14-15 \mathrm{~min}$ while monitoring the reaction with the 
<smiles>[2H]c1cc(/C=C/C(=O)O)cc(C(=O)O)c1N</smiles>

$\alpha$-arylidine- $\beta$-benzoyl propionic acid (4)

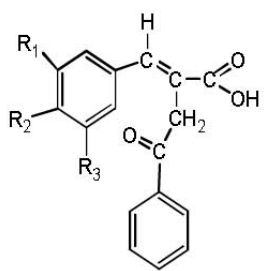

$\alpha$-arylidine- $\beta$-benzoyl propionic acid (4)<smiles>[R]c1cc(/C=C/C(=O)O)cc([2H])c1O</smiles>

$\alpha$-arylidine- $\beta$-benzoyl propionic acid<smiles>[R]c1cc(/C=C(/C(=C)O)c2ccccc2)cc(Br)c1[2H]</smiles>

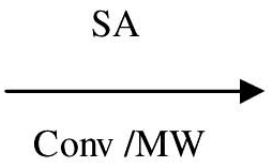

$\alpha$-arylidine- $\beta$-methylene

$\beta$-benzoyl propionic acid (5)<smiles>[R]c1cc(/C=C(/C(=C)O)C(=O)c2ccccc2)cc([R9])c1[2H]</smiles>

Methyl - $\alpha$-arylidine - $\beta$-benzoyl propionic acid (6)

\section{Substitutions}
a: $\mathrm{R}_{1}=\mathrm{R}_{2}=\mathrm{OCH}_{3}, \mathrm{R}_{3}=\mathrm{H}$
b: $\mathrm{R}_{1}=\mathrm{R}_{2}=\mathrm{O}-\mathrm{CH}_{2}-\mathrm{O}, \mathrm{R}_{3}=\mathrm{H}$
c: $\mathrm{R}_{1}=\mathrm{OCH}_{3}, \mathrm{R}_{2}=\mathrm{OH}, \mathrm{R}_{3}=\mathrm{H}$
d: $\mathrm{R}_{1}=\mathrm{R}_{2}=\mathrm{R}_{3}=\mathrm{OCH}_{3}$

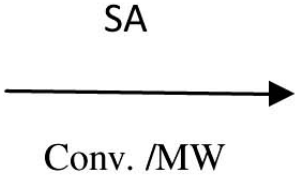

Scheme-II<smiles>[R]c1cc(/C=C(/C(=C)O)C(=O)O)cc([R])c1[2H]</smiles>

$\alpha$-arylidine-

$\beta$-methylene - $\beta$-benzoyl propionic acid (5)<smiles>[R]c1cc(/C=C(/C(=C)Oc2ccccc2)C(C)=O)cc([2H])c1[R]</smiles>

methyl- $\alpha$-arylidine- $\beta$-benzoyl propionic acid (6)<smiles>[R1]c1cc2c(C3CCCCC3)cc(C(=O)O)cc2cc1[18F]</smiles>

1-Phenylnaphthoic acid (7)<smiles>C=C1CCc2c1cc1cc(Cl)c(N)c(O)c1c2-c1ccccc1</smiles>

1-Phenylnaphthalene lactone (8)

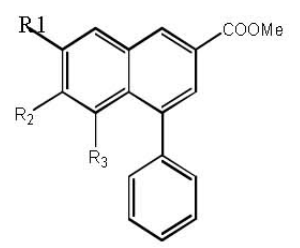

1-Phenylnaphthoate (9) 
TABLE-1

COMPARATIVE DATA ON CONVENTIONAL ACID AND GREEN ACID CATALYZED SYNTHESIS OF 1-PHENYLNAPHTHALENE SYSTEMS

\begin{tabular}{|c|c|c|c|c|c|c|c|c|c|c|c|}
\hline \multirow{3}{*}{ Entry } & \multirow{3}{*}{ Products $^{\mathrm{a}}$} & \multicolumn{4}{|c|}{ Time $(\min )$} & \multicolumn{4}{|c|}{ Yield $(\%)$} & \multirow{2}{*}{\multicolumn{2}{|c|}{ m.p. $\left({ }^{\circ} \mathrm{C}\right)$}} \\
\hline & & \multirow{2}{*}{$\begin{array}{c}\text { PPA } \\
\text { OB } \\
\end{array}$} & \multirow{2}{*}{$\begin{array}{c}\mathrm{H}_{2} \mathrm{SO}_{4} \\
\mathrm{CC}\end{array}$} & \multicolumn{2}{|c|}{ Green-sulphamic acid } & \multirow{2}{*}{$\begin{array}{c}\text { PPA } \\
\text { OB } \\
\end{array}$} & \multirow{2}{*}{$\begin{array}{c}\mathrm{H}_{2} \mathrm{SO}_{4} \\
\mathrm{CC} \\
\end{array}$} & \multicolumn{2}{|c|}{ Green-sulphamic acid } & & \\
\hline & & & & MS & MW & & & MS & MW & Found & Lit. \\
\hline 1 & $7 \mathbf{a}$ & 60 & 1440 & 90 & 15 & 85 & 85 & 93 & 96 & $215-217$ & $217-218^{[12]}$ \\
\hline 2 & $7 b$ & 60 & 1440 & 88 & 14.5 & 84 & 85 & 93 & 95 & $238-240$ & $238-239^{[12]}$ \\
\hline 3 & $7 c$ & 60 & 1440 & 90 & 15 & 78 & 78 & 85 & 85 & $177-179$ & $179-180^{[12]}$ \\
\hline 4 & 7d & 60 & 1440 & 88 & 14.5 & 82 & 81 & 89 & 96 & $220-223$ & $221-223^{[12]}$ \\
\hline 5 & $\mathbf{8 a}$ & 60 & 1440 & 90 & 15 & 82 & 80 & 95 & 94 & $203-205$ & $203-205^{[12]}$ \\
\hline 6 & $8 b$ & 60 & 1440 & 88 & 14.5 & 83 & 83 & 88 & 92 & $209-211$ & $208-209^{[12]}$ \\
\hline 7 & $8 c$ & 60 & 1440 & 90 & 15 & 78 & 78 & 86 & 89 & $209-210$ & $209-210^{[14]}$ \\
\hline 8 & 8d & 60 & 1440 & 89 & 14 & 84 & 82 & 92 & 95 & $233-235$ & $231-233^{[14]}$ \\
\hline 9 & $9 \mathbf{a}$ & 60 & 1440 & 90 & 15 & 80 & 79 & 92 & 94 & $122-124$ & $123-124^{[14]}$ \\
\hline 10 & $9 b$ & 60 & 1440 & 89 & 14.5 & 83 & 83 & 90 & 92 & $170-171$ & $169-171^{[14]}$ \\
\hline 11 & $9 \mathrm{c}$ & 60 & 1440 & 88 & 14.5 & 82 & 82 & 91 & 95 & $150-152$ & $152-153^{[14]}$ \\
\hline 12 & 9d & 60 & 1440 & 89 & 15 & 83 & 83 & 91 & 94 & $130-132$ & $130-132^{[14]}$ \\
\hline
\end{tabular}

${ }^{a}$ Yields refer to pure isolated products; $\mathrm{OB}=$ Oil bath; $\mathrm{CC}=$ Cold condition; $\mathrm{MS}=$ Magnetic stirrer; $\mathrm{MW}=$ Microwave.

TABLE-2

CHARACTERIZATION AND SPECTRAL ANALYSIS OF 1-PHENYLNAPHTHALENE SYSTEMS

\begin{tabular}{|c|c|c|c|c|c|c|}
\hline \multirow{2}{*}{$\begin{array}{l}\text { Sr. } \\
\text { No. }\end{array}$} & \multirow{2}{*}{ 1-Phenylnaphthalene system } & \multirow[t]{2}{*}{ m.f. } & \multicolumn{2}{|c|}{$\begin{array}{l}\text { Elemental analysis } \\
(\%): \text { Obs. (calcd.) }\end{array}$} & \multirow[t]{2}{*}{$\operatorname{IR}\left(\mathrm{cm}^{-1}\right)$} & \multirow[t]{2}{*}{ NMR $(\delta)$} \\
\hline & & & $\mathrm{C}$ & $\mathrm{H}$ & & \\
\hline 1 & $\begin{array}{l}\text { 7a (1-Phenyl -6,7-dimethoxy } \\
\text { naphthalene 3-carboxylic acid) }\end{array}$ & $\mathrm{C}_{19} \mathrm{H}_{16} \mathrm{O}_{4}$ & $\begin{array}{c}74.75 \\
(74.02)\end{array}$ & $\begin{array}{c}5.24 \\
(5.19)\end{array}$ & 1680 & $\begin{array}{l}3.84\left(\mathrm{~s}, 6 \mathrm{H}, 20 \mathrm{CH}_{3}\right), 7.2\left(\mathrm{~s}, 1 \mathrm{H}, \mathrm{C}_{8}-\mathrm{H}\right), 7.35\left(\mathrm{~s}, 1 \mathrm{H}, \mathrm{C}_{5}-\right. \\
\mathrm{H}), 7.43-7.85(5 \mathrm{H} \text {, Phenyl }) \& 8.45\left(\mathrm{~s}, 1 \mathrm{H}, \mathrm{C}_{4}-\mathrm{H}\right)\end{array}$ \\
\hline 2 & $\begin{array}{l}\text { 7b (1-Phenyl-6,7-methylenedioxy } \\
\text { naphthalene-3-carboxylic acid) }\end{array}$ & $\mathrm{C}_{19} \mathrm{H}_{12} \mathrm{O}_{4}$ & $\begin{array}{c}75.24 \\
(75.00)\end{array}$ & $\begin{array}{c}3.96 \\
(3.94)\end{array}$ & 1680 & - \\
\hline 3 & $\begin{array}{l}\text { 7c (1-Phenyl-6-methoxy,7- } \\
\text { hydroxy-naphthalene-3- } \\
\text { carboxylic acid) }\end{array}$ & $\mathrm{C}_{18} \mathrm{H}_{14} \mathrm{O}_{4}$ & $\begin{array}{c}74.22 \\
(73.46)\end{array}$ & $\begin{array}{c}4.81 \\
(4.46)\end{array}$ & 1681 & $\begin{array}{l}4.1\left(\mathrm{~s}, 10 \mathrm{CH}_{3}\right), 4.5(\mathrm{~s}, 1 \mathrm{H}, \mathrm{OH} \text { aromatic protons }), 7.0 \\
\left(\mathrm{~s}, 1 \mathrm{H}, \mathrm{C}_{8}-\mathrm{H}\right), 7.0-8.8(\mathrm{~s} \text {, aromatic protons }), 7.5(\mathrm{~m}, 5 \mathrm{H} \text {, } \\
\text { Phenyl) }\end{array}$ \\
\hline 4 & $\begin{array}{l}\text { 7d (1-Phenyl-6,7,8-trimethoxy } \\
\text { naphthalene-3-carboxylic acid) }\end{array}$ & $\mathrm{C}_{20} \mathrm{H}_{16} \mathrm{O}_{5}$ & $\begin{array}{l}72.07 \\
(71.42)\end{array}$ & $\begin{array}{c}4.80 \\
(4.80)\end{array}$ & 1683 & $\begin{array}{l}3.8-3.9-3.95\left(\mathrm{~s}, 9 \mathrm{H}, 3 \mathrm{OCH}_{3}\right), 6.5-8.5(\mathrm{~m}, \text { aromatic } \\
\text { protons, } 7.5(\mathrm{~m}, 5 \mathrm{H} \text {, phenyl })\end{array}$ \\
\hline 5 & $\begin{array}{l}\text { 8a (1-Phenyl-6,7-dimethoxy } \\
\text { naphthalene lactone) }\end{array}$ & $\mathrm{C}_{20} \mathrm{H}_{16} \mathrm{O}_{4}$ & $\begin{array}{c}75.04 \\
(75.47)\end{array}$ & $\begin{array}{c}5.39 \\
(5.03)\end{array}$ & 1760 & $\begin{array}{l}3.86 \text { \& } 4.06\left(\mathrm{~s}, 6 \mathrm{H}, 2-\mathrm{OCH}_{3}\right) 5.22(\mathrm{~s}, 2 \mathrm{H}, \text { Lactone } \\
\left.\mathrm{CH}_{2}\right), 7.16\left(\mathrm{~s}, 1 \mathrm{H}, \mathrm{C}_{8}-\mathrm{H}\right), 7.37\left(\mathrm{~s}, 1 \mathrm{H}, \mathrm{C}_{5}-\mathrm{H}\right) 7.6(\mathrm{br}, \mathrm{s} \text {, } \\
\text { 5H.phenyl) \& } 8.36\left(\mathrm{~s}, 1 \mathrm{H}, \mathrm{C}_{4}-\mathrm{H}\right)\end{array}$ \\
\hline 6 & $\begin{array}{l}\text { 8b (1-Phenyl-6,7-methylenedioxy } \\
\text { naphthalene lactone) }\end{array}$ & $\mathrm{C}_{19} \mathrm{H}_{12} \mathrm{O}_{4}$ & $\begin{array}{c}75.01 \\
(75.12)\end{array}$ & $\begin{array}{c}3.87 \\
(3.94)\end{array}$ & 1762 & $\begin{array}{l}5.23,\left(\mathrm{~s}, 2 \mathrm{H} \text { lactone } \mathrm{CH}_{2}\right), 6.10\left(\mathrm{~s}, 2 \mathrm{H}, \mathrm{O}-\mathrm{CH}_{2} \mathrm{O}-\right) 7.13- \\
7.4,(\mathrm{~d}, 6 \mathrm{H} \text {, aromatic }), 8.28\left(\mathrm{~s}, 1 \mathrm{H}, \mathrm{C}_{4}-\mathrm{H}\right)\end{array}$ \\
\hline 7 & $\begin{array}{l}\text { 8c (1-Phenyl-6-methoxy-7- } \\
\text { hydroxy-naphthalene lactone) }\end{array}$ & $\mathrm{C}_{20} \mathrm{H}_{16} \mathrm{O}_{4}$ & $\begin{array}{c}75.02 \\
(75.00)\end{array}$ & $\begin{array}{c}5.01 \\
(5.08)\end{array}$ & 1761 & $\begin{array}{l}3.83,\left(\mathrm{~s}, 3 \mathrm{H}, 10 \mathrm{CH}_{3}\right), 5.20\left(\mathrm{~s}, 2 \mathrm{H}, \text { lactone } \mathrm{CH}_{2}\right), 7.04- \\
7.30(\mathrm{~m}, 7 \mathrm{H}, 1 \mathrm{H}, \mathrm{OH} \text { aromatic }), 8.25\left(\mathrm{~s}, 1 \mathrm{H}, \mathrm{C}_{4}-\mathrm{H}\right)\end{array}$ \\
\hline 8 & $\begin{array}{l}\text { 8d (1-Phenyl-6,7,8-trimethoxy } \\
\text { naphthalene lactone) }\end{array}$ & $\mathrm{C}_{21} \mathrm{H}_{19} \mathrm{O}_{5}$ & $\begin{array}{c}71.48 \\
(71.79)\end{array}$ & $\begin{array}{c}5.70 \\
(5.41)\end{array}$ & 1762 & $\begin{array}{l}3.90,4.01,4.25\left(\mathrm{~s}, 9 \mathrm{H}, 3-\mathrm{OCH}_{3}\right), 5.30(\mathrm{~s}, 2 \mathrm{H} \text {, } \\
\left.\text { lactone } \mathrm{CH}_{2}\right), 7.1-7.7(\mathrm{~m}, 6 \mathrm{H} \text {, aromatic }), 8.4\left(\mathrm{~s}, 1 \mathrm{H}, \mathrm{C}_{4}-\right. \\
\mathrm{H})\end{array}$ \\
\hline 9 & $\begin{array}{l}\text { 9a (1-Phenyl-3-carbomethoxy- } \\
6,7 \text {-dimethoxy naphthoate) }\end{array}$ & $\mathrm{C}_{20} \mathrm{H}_{18} \mathrm{O}_{4}$ & $\begin{array}{c}74.14 \\
(74.52)\end{array}$ & $\begin{array}{c}5.52 \\
(5.88)\end{array}$ & 1720 & $\begin{array}{l}3.85,3.96,4.03\left(\mathrm{~s}, 9 \mathrm{H}, \mathrm{CO}_{2}, \mathrm{CH}_{3}, \mathrm{OCH}_{3}\right), 7.24 \& 7.30 \\
\left.(\mathrm{~s}, \mathrm{sh}) \mathrm{C}_{3}-\mathrm{H} \& \mathrm{C}_{8}-\mathrm{H}\right), 7.85(\mathrm{~s}, \mathrm{sh}, \text { phenyl), } 7.9(\mathrm{~d}, J= \\
\left.2 \mathrm{H}_{2}, 1 \mathrm{H}, \mathrm{C}_{4}-\mathrm{H}\right), 8.47\left(\mathrm{~d}, J=2 \mathrm{H}_{2}, \mathrm{C}_{2}-\mathrm{H}\right)\end{array}$ \\
\hline 10 & $\begin{array}{l}\text { 9b (1-Phenyl-3-carbomethoxy- } \\
6,7 \text {-methylenedioxy naphthoate) }\end{array}$ & $\mathrm{C}_{21} \mathrm{H}_{14} \mathrm{O}_{4}$ & $\begin{array}{l}76.19 \\
(76.36)\end{array}$ & $\begin{array}{c}4.12 \\
(4.24)\end{array}$ & 1720 & $\begin{array}{l}4.02\left(\mathrm{~s}, 3 \mathrm{H}, \mathrm{OCH}_{3}\right), 5.94\left(\mathrm{~s}, 2 \mathrm{H}, \mathrm{OCH}_{2} \mathrm{O}-\right), 7.32 \& 7.38 \\
\left(5(\mathrm{sh}) 2 \mathrm{H}, \mathrm{C}_{5}-\mathrm{H} \& \mathrm{C}_{8}-\mathrm{H}\right), 7.60(\mathrm{~s}, \mathrm{sh}, \text { phenyl }), 8.04(\mathrm{~d}, \\
\left.J=2 \mathrm{H}_{2}, 1 \mathrm{H}, 2 \mathrm{H}\right) \text { and } 8.86\left(\mathrm{~d}, J=2 \mathrm{H}_{2}, 1 \mathrm{H}, \mathrm{C}_{4}-\mathrm{H}\right)\end{array}$ \\
\hline 11 & $\begin{array}{l}\text { 9c (1-Phenyl-6-methoxy-7- } \\
\text { hydroxy-naphthoate) }\end{array}$ & $\mathrm{C}_{19} \mathrm{H}_{18} \mathrm{O}_{4}$ & $\begin{array}{c}73.38 \\
(73.54)\end{array}$ & $\begin{array}{c}5.68 \\
(5.80)\end{array}$ & 1720 & - \\
\hline 12 & $\begin{array}{l}\text { 9d (1-Phenyl-3-carbomethoxy- } \\
6,7,8 \text {-trimethoxy naphthoate) }\end{array}$ & $\mathrm{C}_{21} \mathrm{H}_{22} \mathrm{O}_{5}$ & $\begin{array}{c}71.02 \\
(71.18) \\
\end{array}$ & $\begin{array}{c}6.05 \\
(6.21) \\
\end{array}$ & 1720 & - \\
\hline
\end{tabular}

help of TLC. After the completion of the reaction, immediate temperature of the reaction mixture was taken out by the thermometer, which is recorded as $75^{\circ} \mathrm{C}$.The reaction was worked -up and purified to give 1-phenyl-naphthoate. Adopting the above described two alternative methods, four different substituted 1-phenylnaphthoates (9a-9d) were identified and characterized (Table-2).

\section{RESULTS AND DISCUSSION}

To prepare 1-phenylnaphthalene system and pericarbonyl lactone lignans, $\beta$-benzoyl propionic acid ${ }^{16}$ was used which has two reactive methylene groups and a carboxylic functional group which could lead to the basic skeleton of lignans. The carboxyl group would yield part of furan ring and the oxo 

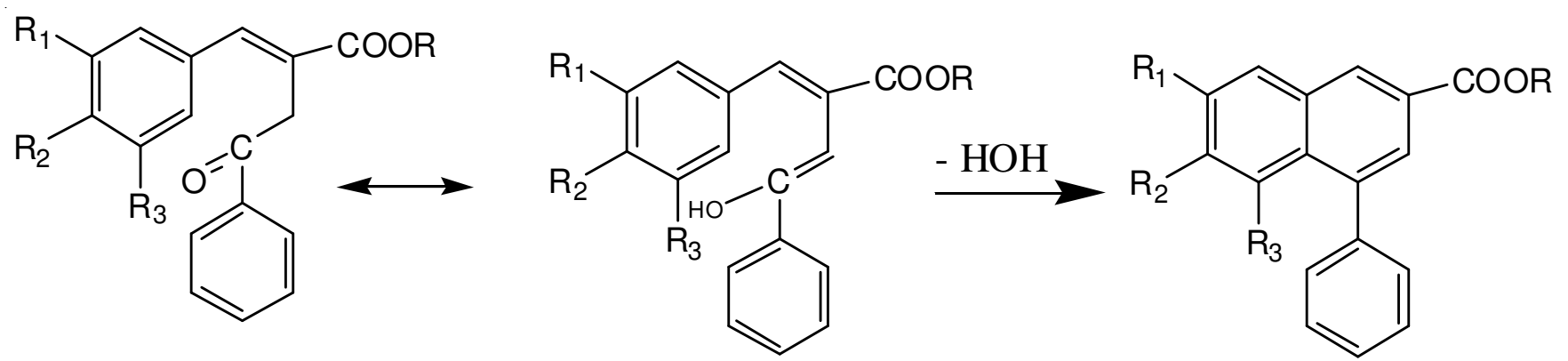

Scheme-III

group could be reduced. $\beta$-Benzoyl propionic acid with aryl aldehydes underwent Perkin condensation at $\alpha$-methylene to give $\alpha$-arylidine- $\beta$-benzoyl propionic acid (Scheme-I). This condensation reaction is restricted to aldehydes having hydroxyl, methoxy or methylenedioxy groups at para positions only ${ }^{17}$. Further cyclization of Perkin condensation product- $\alpha$-arylidine$\beta$-benzoyl propionic acid using sulphamic acid gives 1-phenylnaphthalene system and pericarbonyl lactone lignans under two experimental conditions-conventional and microwave (Scheme-II).

To extend the scope of reaction and to generalize the procedure, we carried out the reaction of $\beta$-benzoyl propionic acid with a series of aryl aldehydes (veratraldehyde, pipernol, vanillin, 3,4,5-trimethoxy-benzaldehyde) to obtain the corresponding 1-phenylnaphthalene systems 7a-d, 8a-d and 9a-d by adopting the above described two alternative methods. All the cyclized products have been characterized by their IR, ${ }^{1} \mathrm{H}$ NMR spectral values and elemental analysis (Table-2).

The synthesis of (4) to (7) and (6) to (9) takes place involving keto-enol tautomerism followed by removal of $\mathrm{H}$ and $\mathrm{OH}$. While in system (5) ( $\alpha$-arylidine- $\beta$-methylene- $\beta$-benzoyl propionic acid) there is hyper-conjugation at $\mathrm{CH}_{2}$ and cyclization take place by keto-enol tautomerism. The aromatic atmosphere spread is more on S-cis butadiene than S-trans orientation. Though the S-trans is more stable, here $\mathrm{S}$-cis has more chance to exit. Hence the facile cyclization via keto-enol tautomerization which in turn existed due to hyper conjugation which is possible due to methylene group. All these intermediate structures drive the reaction towards aryl lactonization via cyclization by removal of $\mathrm{H}$ and $\mathrm{OH}$ and simultaneous tautomerism at the $\mathrm{COOH}$ and positive $\mathrm{CH}_{2}$ positive centre which leads to the formation of pericarbonyl lactone.

The literature survey disclose plethora of cyclizing reagents such as $\mathrm{H}_{2} \mathrm{SO}_{4}$, polyphosphoric acid ${ }^{18}, \mathrm{CH}_{3} \mathrm{COOH} /$ $\mathrm{HCl}^{19,20}$, lead tetra acetate ${ }^{21}$ for the synthesis of 1-phenylnaphthalene system. However, processes involving conventional acids are inherently associated with problems such as high toxicity, corrosion, catalyst waste, difficulty in separation and recovery. Replacement of these conventional acids by solid catalyst is desirable to achieve effective catalyst handling, product purification and to decrease waste production.

Also the above conventional cyclization reactions have been done using traditional heat transfer equipments such as oil-bath, sand bath and heating jackets. These heating techniques are however, rather slow and a temperature gradient can develop. Therefore it is seen that above cyclization reactions have been plagued by a number of serious disadvantages. Now we have found an analogous cyclization reaction which can be conveniently performed under neutral and mild conditions in the presence of catalytic amount of sulphamic acid under the cooperative effect of microwave radiation and a novel conventional refluxing/stirring method.

When compared to other acid catalysts, the use of sulphamic acid facilitated the smooth conduct of the cyclization reactions. The work out of the product was very simple and better yields were recorded. As expected, the alternative microwave-assisted method was found to be more convenient over that of conventional, in terms of considerable reduction in reaction time, improved yields and facile nature of reaction.

In this method the use of $20 \mathrm{~mol} \%$ of the catalyst was quite sufficient to promote the reaction; higher amount of the catalyst did not improve the yield. The use of sub-stoichiometric quantity of sulphamic acid indicated its true catalytic nature and also gave an advantage over other classical Bronsted acids. Recovery of catalyst was very easy so that the sulphamic acid could be recycled upto three cycles without significant loss in its activity (Table-3).

\begin{tabular}{ccc|cc|cc}
\hline \multicolumn{8}{c}{ TABLE-3 } \\
\multicolumn{7}{c}{$\begin{array}{c}\text { RECYCLABILITY OF SULPHAMIC ACID } \\
\text { IN THE CYCLIZATION REACTIONS }\end{array}$} \\
\hline Cycle & \multicolumn{2}{c}{ Yield (\%) 7a } & \multicolumn{2}{c}{ Yield (\%) 8a } & \multicolumn{2}{c}{ Yield (\%) 9a } \\
\cline { 2 - 7 } No. & Conv. & MW & Conv. & MW & Conv. & MW \\
\hline Fresh & 93 & 96 & 95 & 94 & 92 & 94 \\
1 & 92 & 95 & 94 & 93 & 91 & 92 \\
2 & 90 & 94 & 92 & 91 & 90 & 91 \\
3 & 88 & 92 & 91 & 90 & 88 & 88 \\
\hline${ }^{a}$ Isolated yield.
\end{tabular}

\section{Conclusion}

In summary, we have developed a simple and general method for the synthesis of 1-phenylnaphthalene system and pericarbonyl lactone lignans by using sulphamic acid as a recyclable heterogeneous catalyst under the cooperative effect of microwave radiation and a novel conventional method. The procedure is convenient and highly efficient since the titled compounds are produced in good to excellent yields after shot reaction times. Moreover, sulphamic acid showed high thermal stability and can be recovered and reused for at least three consecutive cycles without significant loss of its activity. Consequently, our method can be as a viable alternative to the presently existing procedures. 


\section{ACKNOWLEDGEMENTS}

The authors are thankful to Dr. M.T. Bharambe, Director, Institute of Science, Nagpur, for providing the necessary research facilities. Thanks are also due to SAIF, Chandigarh for spectral facilities.

\section{REFERENCES}

1. H.S. Ban, S. Lee, Y.P. Kim, K.Yamaki, K.H. Shin and K. Ohuchi, Biochem. Pharmacol., 64, 1345 (2002).

2. K. Murakamiand and A. Tomasz, J. Bacteriol., 171, 874 (1989).

3. N.Y. Mono, J.C. Masuoka, A. Liokawa, M. Tikeda and Tnohara, J. Nat. Prod., 67, 2073 (2004).

4. S. Deo, F. Inam and A.N. Jadhav, Asian J. Chem., 24, 2622 (2012).

5. E. Navarro, S.J. Alonso, P.J. Alonso, J. Trujillo, E. Jorge and C. Perez, Biol. Pharm. Bull., 24, 254 (2001).

6. T.S. Jin, G. Sun, Y.W. Li and T.S. Li, Green Chem., 4, 255 (2002)

7. W. Bo, Y.L. Ming and S.J. Shuan, Tetrahedron Lett., 44, 5037 (2003).

8. B. Wang, Y.L. Gu, C. Luo, T. Yang, L.M. Yang and J.S. Suo, Tetrahedron Lett., 45, 3369 (2004).

9. P.R. Singh, D.U. Singh and S.D. Samant, Synlett, 1909 (2004).
10. M.M. Heravi, L. Ranjbar, F. Derikvand and B. Alimadadi, Mol. Divers., 12, 191 (2008).

11. A.I. Scott, Interpretation of the UV Spectra of Natural Products, Pergamon Press, London, p. 7 (1964).

12. F. Rashedian, D. Saberi and K. Niknam, J. Chin. Chem. Soc., 57, 998 (2010).

13. A.A. Avetisyan and G.G. Tokmadzhyan, Chem. Heterocycl. Compd., 23, 595 (1986).

14. P.B. Simon, J.Y. Oveden and S.S. Wan, Phytochemistry, 65, 2977 (2004).

15. W.F. Fong, A.K.W. Tse, K.H. Poon and C. Wang, Intl. J. Biochem. Cell Biol., 37, 427 (2005).

16. B.S. Furniss, A.J. Hannaford, P.W.G. Smith and A.R. Tatchell, Vogel's Textbook of Practical Organic Chemistry; Harlow, Longman, edn. 5 (1989).

17. G.K. Bradsher, Chem. Rev., 38, 465 (1946).

18. S. Deo, F. Inam, R.P. Mahashabde and A.N. Jadhav, Asian J. Chem., 22, 3362 (2010).

19. P.W. Agnihotri, V.R. Pasarkar and G. Bagawant, J. Indian Chem. Soc., 59, 869 (1982).

20. R. Stevenson, Stud. Nat. Prod. Chem., 17, 311 (1995).

21. R.D. Haworth and D. Woodcock, J. Chem. Soc., 154 (1939). 